\title{
Multivariate analysis of risk factors for ESBL and AmpC producing Escherichia coli and Klebsiella pneumoniae at a Tertiary Care Hospital in Karnataka: A case control study
}

\author{
Aaftab G.P1, Asha B Patil ${ }^{2 *}$, Shobha Medegar ${ }^{3}$ \\ ${ }^{\mathbf{1}}$ Assistant Professor, Dept. of Microbiology, Navodaya Medical College Hospital, Raichur, Karnataka, ${ }^{2}$ Professor \& HOD, \\ ${ }^{3}$ Tutor, Dept. of Micribiology, Karnataka Institute of Medical College, Hubballi, Karnakata, India
}

*Corresponding Author:

Email: drashapatil@ rediffmail.com

\begin{abstract}
Introduction: Extended spectrum $\beta$-lactamase (ESBL) and AmpC producing organisms pose unique challenges to clinical microbiologists, clinicians, infection control professionals.

The objective of the present study was to analyze the risk factors that will help in identifying those at risk of acquiring infection by ESBL and/or AmpC producing organisms thereby making appropriate changes in the choice of antimicrobial therapy and minimizing treatment failures.

Materials and Methods: Risk factors were evaluated using a retrospective Case control design. 100 patients with infections due to ESBL and/ or AmpC producing E.coli or K. pneumoniae were employed as cases. These were matched with 100 patients with non ESBL- non AmpC producing organisms (Controls). Univariate and Multivariate logistic regression analysis was performed to identify variables associated with becoming a case.

Results: Out of the total 100 cases, E.coli 61 (61\%) was the predominant ESBL and/or AmpC producer, followed by Klebsiella pneumonia $39(39 \%)$. In the multivariate analysis, we found that duration of hospital stay for more than 5 days $(\mathrm{OR}=0.16, \mathrm{P}=0)$, stay in ICU $(\mathrm{OR}=2.98, \mathrm{P}=0.02)$ and prior treatment with antibiotics $(\mathrm{OR}=2.81, \mathrm{P}=0.003)$ were statistically significant risk factors associated with acquisition of ESBL/AmpC producers. We also found invasive procedures like usage of urinary catheter $(\mathrm{OR}=3.34, \mathrm{P}<0.005)$, central venous catheter $(\mathrm{OR}=3.35, \mathrm{P}=0.018)$, intravenous catheter $(\mathrm{OR}=7.38, \mathrm{P}<0.005)$ and other surgical procedures $(\mathrm{OR}=3.14, \mathrm{P}=0.0002)$ contributed significantly to the spread of ESBL/AmpC producers.

Conclusion: ESBL and AmpC producing strains were found to show higher rates of resistance to various class of antibiotics when compared to non ESBL and AmpC producers. Knowledge on risk factors of infection helps physicians in framing appropriate empirical antibiotic policies.
\end{abstract}

Keywords: AmpC resistance, Case control, Extended spectrum beta lactamases, Risk factors.

\section{Introduction}

Extended Spectrum Beta Lactamases are most often found in strains of Escherichia coli and Klebsiella pneumoniae. ${ }^{1}$ In many parts of the world, $10-40 \%$ of strains of Escherichia coli and Klebsiella pneumoniae express ESBLs. ${ }^{2}$ High rates of AmpC $\beta$-lactamase (ABL) producing Enterobacteriaceae have been reported- $36.5 \%$ in India, 35.6\% in Pakistan and 26.2\% in China. ${ }^{3}$

Infections with ESBL-producing Enterobacteriaceae originally emerged primarily in the hospital setting. Patients at high risk for developing infection with ESBL-producing organisms are often seriously ill, have (had) a prolonged hospital stay and in whom invasive medical devices are present (urinary catheters, endotracheal tubes, central venous lines) for a prolonged duration, have had prior antibiotics or have severe underlying disease..$^{4-8}$

Since the ESBL genes are usually found in large plasmids, they also contain other antimicrobial resistant genes. Therefore most ESBL producing organisms are also resistant to aminoglycosides, fluoroquinolones, tetracyclines, chloramphenicol, sulfonamides. ${ }^{9}$ Their resistance to a wide variety of common antimicrobials has made the proliferation of ESBL producing strains a serious global health concern that has complicated treatment strategies. ${ }^{10}$ Nosocomial infections caused by multidrug resistant (MDR) Gram-negative bacteria expressing ESBL pose serious therapeutic challenge to clinicians due to limited therapeutic options. ${ }^{5}$ It is also imperative that risk factors for infection with ESBL producing organisms be clearly identified so that targeted therapy and effective strategies can be developed to curtail the emergence and spread of these strains. ${ }^{5-8}$

Based on this premise the present study was undertaken to evaluate the risk factors for infections with extended spectrum $\beta$-lactamase and AmpC producing Escherichia coli and Klebsiella species, among patients admitted at Karnataka Institute of Medical Sciences, Hubballi.

\section{Materials and Methods}

Setting and Study design: A case control study was performed to define the risk factors for ESBL and/or AmpC acquisition. Cases were defined as patients who were hospitalised and from whom ESBL and/or AmpC producing E.coli and K.pneumoniae were isolated during the study period. Potential controls were recruited from the cohort of hospitalised patients and 
were defined as those presenting with the same types of infections as those of the cases, but with infections due to non-ESBL non AmpC producing E.coli and K.pneumoniae. Controls were selected randomly from the hospitalised patients with same date of admission and same bacterial isolate. Cases and controls were not otherwise matched.

Data collection and Definitions: Clinical and demographic data of cases and controls were collected from patients' charts .The variables evaluated as possible risk factors were: age, sex, hospital stay of >5days, ICU stay, mechanical ventilation, central venous catheter, urinary catheter prior hospitalisation, prior surgical procedure, prior antibiotic use within 1 year of diagnosis, associated clinical condition or comorbidity, immunosuppressive therapy.

Microbiological analysis: Bacterial isolates of E.coli and Klebsiella pneumonia were subjected to antibiotic susceptibility testing by disc diffusion technique according to CLSI guidelines. ${ }^{11}$

Screening test for ESBL detection: ${ }^{4,11}$ A zone diameter of $<22 \mathrm{~mm}$ for Ceftazidime $30 \mu \mathrm{g}$ disk was taken as Screening test positive for ESBL. All E.coli and Klebsiella pneumoniae which were screening positive were subjected to phenotypic confirmatory tests for ESBL detection.

Phenotypic confirmation test for ESBL detection: ${ }^{4,11}$ Lawn culture of the isolate was made. Ceftazidime disks $(30 \mu \mathrm{g})$ alone and in combination with clavulanate $(10 \mu \mathrm{g})$ were placed at a distance of $20 \mathrm{~mm}$ and incubated for 24 hours at $37^{\circ} \mathrm{C}$. A difference of $\geq 5 \mathrm{~mm}$ between the zone diameters of ceftazidime disk and the ceftazidime-clavulanate combination disk was taken to be confirmatory for ESBL production.

Screening test for Amp-C production: ${ }^{12}$ Isolates were screened for Amp-C production by using Cefoxitin disk $(30 \mu \mathrm{g})$. Isolates with cefoxitin zone of $<18 \mathrm{~mm}$ were considered as screen positives for Amp-C betalactamase production.

Phenotypic confirmatory test for Amp-C betalactamase production: All the Amp-C screen positive isolates were subjected to phenotypic confirmatory test for Amp-C beta lactamase production by Modified three dimensional test.

Modified Three Dimensional Test for AmpC detection: ${ }^{12}$ Fresh overnight growth from Muller Hinton agar (MHA) is transferred to a pre-weighed sterile microcentrifuge tube. The tube is weighed again to determine the weight of bacterial mass to obtain 10$15 \mathrm{mg}$ of bacterial wet weight. The bacterial mass is suspended in peptone water and pelleted by centrifugation at $3000 \mathrm{rpm}$ for 15 minutes. Crude enzyme extract is prepared by repeated freeze-thawing of the bacterial pellet (10 cycles). Lawn culture of Escherichia coli ATCC 25922 is prepared on MHA plates and cefoxitin $(30 \mu \mathrm{g})$ disks are placed on the plates. Linear slits $(3 \mathrm{~cm})$ are cut using sterile surgical blade, $3 \mathrm{~mm}$ away from cefoxitin disk. $30 \mu \mathrm{l}$ of the enzyme extract is loaded into the slit. The plates are kept upright for 5 to 10 minutes until the liquid is dried and are then incubated at $37^{\circ} \mathrm{C}$ for 24 hours. Enhanced growth of the surface organism at the point where the slit intersected the zone of inhibition of cefoxitin, causing a clear distortion to this zone is considered positive three dimensional test and is interpreted as evidence for the presence of Amp-C $\beta$-lactamases. Isolates with no distortion are recorded as non-Amp-C producers.

\section{Statistical analysis}

The results were analysed using two tailed Chisquare test, Fisher's exact test and test of proportions wherever applicable. We used conditional logistic regression analysis for univariate and multivariate calculation of risk factors and odds ratio (OR) with 95\% confidence intervals (CI). Difference in proportion were considered significant if $\mathrm{p}$ was $<0.05$. Analysis was performed using SPSS software version 20.

\section{Results}

A total of 200 patients (100 cases and 100 conttrols) were included in the analysis. 114 (57.0\%) patients were male, of whom 56 were in case patients group and 58 belonged to control patients group. Most (34\%) of the ESBL and/or AmpC producers were isolated from Surgery wards, followed by Medicine (29\%), orthopaedics (16\%) and the ICUs (13\%). ICU admission was more likely to be associated ESBL and/or AmpC producing isolates ( $\mathrm{p}=0.048$ ) according to the multivariate analysis. In our study, among the 100 cases, we isolated 46 pure ESBL producers, 1 pure AmpC producer and 53 Co-producers (ESBL+AmpC).

\section{Risk factors analysis}

Various studies have reported multiple risk factors associated with ESBL and AmpC producing organisms. In present study we analyzed the following risk factors which include gender, duration of hospital stay, stay in ICU, prior treatment with antibiotics, history of immunosuppressive drugs, use of intravenous catheter, urinary catheter, parenteral feeding, mechanical ventilation, tracheostomy, other invasive procedures, immunodeficiency syndrome and any other underlying clinical condition. There were no significant differences in the demographic or risk factor variables collected when comparing ESBL and AmpC cases and therefore the two groups were combined and classified as one, ESBL/AmpC, for the purpose of the present study. 
Table 1: Multivariate analysis of risk factors for infection by ESBL and AmpC producing E.coli and Klebsiella

\begin{tabular}{|c|c|c|c|c|c|c|}
\hline $\begin{array}{c}\text { Risk } \\
\text { factors }\end{array}$ & $\begin{array}{c}\text { ESBL and/or } \\
\text { AmpC } \\
\text { producers } \\
(\mathbf{n}=\mathbf{1 0 0})\end{array}$ & $\begin{array}{c}\text { Non ESBL } \\
\text { and AmpC } \\
\text { producers } \\
(n=100)\end{array}$ & $\begin{array}{l}\text { Odds } \\
\text { ratio }\end{array}$ & $\begin{array}{c}\text { Chi } \\
\text { square } \\
\text { value }\end{array}$ & P value & Significance \\
\hline \multicolumn{7}{|l|}{ Sex } \\
\hline Male & 57 & 57 & 1 & 0 & 1 & NS \\
\hline Female & 43 & 43 & & & & \\
\hline \multicolumn{7}{|c|}{ Duration of Hospital stay } \\
\hline$<5$ days & 19 & 59 & 0.16 & 33.62 & 0 & $S$ \\
\hline$>5$ days & 81 & 41 & & & & \\
\hline \multicolumn{7}{|c|}{ Stay in ICU } \\
\hline Yes & 16 & 06 & 2.98 & 5.10 & 0.023 & $\mathrm{~S}$ \\
\hline No & 84 & 94 & & & & \\
\hline \multicolumn{7}{|c|}{ Prior treatment with antibiotics } \\
\hline Yes & 68 & 43 & 2.81 & 12.65 & 0.003 & $S$ \\
\hline No & 32 & 57 & & & & \\
\hline \multicolumn{7}{|c|}{ History of Immunosuppressive drugs } \\
\hline Yes & 0 & 03 & 0.13 & 3.04 & 0.246 & NS \\
\hline No & 100 & 97 & & & & \\
\hline \multicolumn{7}{|c|}{ Immunodeficiency Syndrome } \\
\hline Yes & 04 & 00 & 9.37 & 4.08 & 0.121 & NS \\
\hline No & 96 & 100 & & & & \\
\hline \multicolumn{7}{|c|}{ Underlying clinical condition } \\
\hline Yes & 25 & 24 & 1.05 & 0.027 & 0.869 & NS \\
\hline No & 75 & 76 & & & & \\
\hline
\end{tabular}

Univariate analysis noted significant differences between cases and controls. Those with $p$ value of $<0.20$ were included in the multivariate analysis. In the multivariate analysis, risk of infection with ESBL and AmpC producing E.coli and Klebsiella species was found to be around 3 times more in patients with ICU stay $(\mathrm{OR}=2.98, \mathrm{p}=0.023)$ and prior treatment of antibiotics $(\mathrm{OR}=2.81, \mathrm{p}=0.003)$. Patients with immunodeficiency syndrome ( $\mathrm{OR}=9.37$, $\mathrm{p}=0.121)$ and underlying clinical condition $(\mathrm{OR}=1.05, \mathrm{p}=0.869)$ also showed increased risk of infection with ESBL and $\mathrm{AmpC}$ with odds ratio more than one. However, statistically significant results were obtained only for duration of hospital stay $>5$ days $(\mathrm{OR}=0.16, \mathrm{p}=0)$, stay in $\mathrm{ICU}(\mathrm{OR}=2.98, \mathrm{p}=0.023)$ and prior treatment of antibiotics $(\mathrm{OR}=$ $2.81, \mathrm{p}=0.003)$.

Table 2: Multivariate analysis of risk factors for infection by ESBL and AmpC producing E.coli and Klebsiella, taking invasive procedures into consideration

\begin{tabular}{|l|c|c|c|c|c|c|}
\hline $\begin{array}{c}\text { Risk factors } \\
\text { (Invasive } \\
\text { procedures) }\end{array}$ & $\begin{array}{c}\text { ESBL } \\
\text { and/or } \\
\text { AmpC } \\
\text { producers } \\
\text { (n=100) }\end{array}$ & $\begin{array}{c}\text { Non ESBL } \\
\text { and AmpC } \\
\text { producers } \\
\text { (n=100) }\end{array}$ & $\begin{array}{c}\text { Odds } \\
\text { ratio }\end{array}$ & $\begin{array}{c}\text { Chi } \\
\text { square } \\
\text { value }\end{array}$ & P Value & Significance \\
\hline Urinary catheter & 50 & 23 & 3.34 & 15.72 & $7.3 \mathrm{E}-05$ & $\mathrm{~S}$ \\
\hline Yes & 50 & 77 & & & & \\
\hline No & 57 & 3.35 & 5.55 & 0.018 & $\mathrm{~S}$ \\
\hline Central venous catheter \\
\hline Yes & 15 & 05 & & & & \\
\hline No & 85 & 95 & & & \\
\hline Intravenous catheter & 95 & 72 & 7.38 & 19.19 & $1.2 \mathrm{E}-05$ & $\mathrm{~S}$ \\
\hline Yes & 95 & 28 & & & & \\
\hline No & 05 & &
\end{tabular}




\begin{tabular}{|l|c|c|c|c|c|c|}
\hline Yes & 15 & 06 & 2.76 & 4.30 & 0.062 & NS \\
\hline No & 85 & 94 & & & & \\
\hline Tracheostomy & 01 & 00 & 3.03 & 1.005 & 0.316 & NS \\
\hline Yes & 99 & 100 & & & & \\
\hline No & & & & & \\
\hline \\
\hline Other Invasive procedures \\
\hline Yes & 44 & 20 & 3.14 & 13.23 & 0.0002 & S \\
\hline No & 56 & 80 & & & & \\
\hline
\end{tabular}

Risk of infection with ESBL and AmpC producing E.coli and Klebsiella species was found to be around 3 times more in patients with urinary catheter $(\mathrm{OR}=3.34, \mathrm{p}=7.3 \mathrm{E}-05)$ and central venous catheter $(\mathrm{OR}=3.35, \mathrm{p}=0.018) ; 7$ times more in patients with intravenous catheter $(\mathrm{OR}=7.38, \mathrm{p}=1.2 \mathrm{E}-05)$. Patients with Tracheostomy $(\mathrm{OR}=3.03$, $\mathrm{p}=0.316$ ) also showed increased risk of infection with ESBL and AmpC with odds ratio more than one. However, statistically significant results were obtained only for use of urinary catheter, central venous catheter, intravenous catheter $(\mathrm{OR}=7.38, \mathrm{p}=1.2 \mathrm{E}-05)$ and invasive procedures like laparotomy, appendicectomy and other surgical procedures $(\mathrm{OR}=3.14, \mathrm{p}=0.0002)$.

Prior antibiotic use: Table 3 depicts the antibiotic treatment history of cases and controls within 1 year preceding the onset of infection.

Table 3: History of antibiotics received among ESBL/AmpC producers and the non ESBL/AmpC group prior to onset of infection.

\begin{tabular}{|l|c|c|c|c|}
\hline \multicolumn{1}{|c|}{ Antibiotic } & $\begin{array}{c}\text { ESBL/AmpC } \\
\text { group, } \mathbf{n = 1 0 0}(\boldsymbol{\%})\end{array}$ & $\begin{array}{c}\text { Non- } \\
\text { ESBL/AmpC } \\
\text { group, } \mathbf{n = 1 0 0 ( \% )}\end{array}$ & p- value & Significance \\
\hline Penicillins & 10 & 3 & 0.058 & NS \\
\hline $\begin{array}{l}3^{\text {rd }} \text { generation } \\
\text { cephalosporins }\end{array}$ & 63 & 37 & 0.0003 & $\mathrm{~S}$ \\
\hline $\begin{array}{l}4^{\text {th }} \text { generation } \\
\text { cephalosporins }\end{array}$ & 02 & 00 & 0.294 & NS \\
\hline Quinolones & 27 & 18 & 0.129 & NS \\
\hline Aminoglycosides & 41 & 17 & 0.0003 & S \\
\hline Azithromycin & 01 & 01 & 1 & NS \\
\hline
\end{tabular}

A significant increase in number of infections due to ESBL/AmpC producing bacteria was found in patients with a history of previous treatment with $3^{\text {rd }}$ generation cephalosporins (63\% vs $37 \%, \mathrm{p}=0.0003)$ and aminoglycosides ( $41 \%$ vs $17 \%, \mathrm{p}=0.0003)$. Contrary to our belief, quinolones usage ( $27 \%$ vs $18 \%, \mathrm{p}=0.129)$ was found to be statistically not significant.

\section{Discussion}

The incidence of infections due to organisms resistant to $\beta$ lactam agents has increased sharply in recent years. ${ }^{8}$ It is imperative that risk factors for infections due to ESBL-producing organisms be clearly identified so that effective strategies to limit outbreaks of these infections may be developed. ${ }^{8}$ Past attempts to identify risk factors for infection due to ESBLproducing organisms have come to very different conclusions. This fact may be partly because most previous studies failed to distinguish colonization with such pathogens from true infection. Although early correlational studies suggested an association between antimicrobial use and the emergence of ESBL- producing E.coli or K.pneumoniae infections, these studies failed to control for confounding factors and lacked comparison groups.

We performed a Case-control study comparing 100 patients with infections due to ESBL/AmpC-producing Escherichia coli or Klebsiella pneumoniae vs 100 controls with infections due to non-ESBL/AmpC producing organisms in which we identified the following major risk factors: Longer duration of hospital stay, stay in ICU, prior treatment with antibiotics, urinary catheter, central venous catheter, intravenous catheter, invasive procedures.

Prior treatment with antibiotics was identified as a significant risk factor for infection with ESBL producing strains in different studies done by Santhi M et al. ${ }^{[5]}$, Pena $\mathrm{C}$ et al., ${ }^{1}$ Baral $\mathrm{P}$ et al., ${ }^{3}$ Ozgunes I et al. ${ }^{13}$ and Tumbarello $\mathrm{M}$ et al. ${ }^{9}$ It was correlating with our present study. Similarly longer duration of hospital stay was identified as independent risk factor in various studies conducted by Santhi M et al., ${ }^{5}$ Baral $\mathrm{P}$ et $\mathrm{al}^{3}{ }^{3}$ and Tumbarello $\mathrm{M}$ et al. ${ }^{9}$ Furthermore, Foley's catheter, intravenous catheter, central venous catheter, 
intubation, surgery and mechanical ventilation were identified as risk factors associated with ESBL production by the study done by Ozgunes I et al. ${ }^{13}$ which correlated with our study.

The role of antibiotics use has been emphasized as the leading risk factor associated with ESBL producing organisms ${ }^{14}$ and this finding is confirmed by our multivariate analysis. Empirical antibiotic therapy promotes colonization in hospitalised patients with resistant strains by eradicating susceptible flora. ${ }^{5}$ Eradication or reduction of drug susceptible normal flora can also increase one's vulnerability to the acquisition of new strains. This effect can increase the risk of infection by resistant organisms if exposure occurs during or shortly after antibiotic treatment. In addition, an organism that is resistant to multiple drugs may be more subject to selection by the use of any of those drugs. ${ }^{9}$ They are the reservoirs of infection and facilitate the persistence of multidrug resistant bacteria in hospital. ${ }^{5}$

The use of third generation cephalosporins has been reported as the most important class in some studies. In addition to third generation cephalosporins, quinolones, aminoglycosides and carbapenems have also been accused..$^{14}$ Our results support the hypothesis that there is strong association of previous antibiotic use with infections caused by ESBL-producing organisms. In the present study, 68 patients out of 100 cases admitted, gave history of prior treatment with antibiotics, whereas there were only 43 patients out of 100 controls who had been on antibiotics previously. Futhermore, we found only the previous use of thirdgeneration cephalosporins $(\mathrm{p}=0.003)$ and aminoglycosides $(\mathrm{p}=0.003)$ as significant risk factors. Contrary to various other studies conducted, quinolones were not found to be significant risk factors in this study.

Third generation cephalosporins represent the most commonly used class of antibiotics in hospitals, therefore exerting a predominant selective pressure for development of resistance. Our result confirms the fact that ESBLs may emerge as a result of excessive cephalosporin use, and also indicates that interventions designed to restrict cephalosporin use in order to reduce the level of antibiotic resistance merits further investigation. A study conducted by M Shanthi and Uma Sekar ${ }^{5}$ at Chennai, India concluded that prior exposure to multiple antibiotics especially beta lactam antibiotics was an important contributing factor in acquisition of ESBL producing infections. Pena $\mathrm{C}$ et al ${ }^{1}$ in their study at Spain found previous antibiotic therapy $(\mathrm{OR}=3.9, \mathrm{p}<0.001)$ as one of the independent variables associated with ESBL producing organisms.

Longer duration of hospital stay ( $>5$ days) was identified as a significant risk factor in the present study. This was in accordance with the studies done by Santhi M et al, ${ }^{10}$ Lautenbach E et al,${ }^{8}$ Tumbarello $M$ et $\mathrm{al}^{9}$ and Agostini $\mathrm{M}$ et al. ${ }^{15}$ Also, ESBL/AmpC producing infections contribute to prolonged hospital stay and increased antibiotic usage eading ultimately to economic burden in hospitalised patients. Detecting the presence of ESBL/AmpC producing pathogens in specimens obtained from patients has important implications for clinical decision making, chiefly by influencing the choice of appropriate therapy. In addition to their clinical value, their detection can also aid in infection control measures by helping to guide patient isolation strategies. ${ }^{10}$

Numerous studies have used a case-control design with which to assess risk factors for colonization and infection with ESBL/AmpC-producing organisms. ${ }^{5,1,14,13,16}$ Analysis of the results of these studies yields a plethora of conflicting results, likely due to differences in study populations, selection of cases, selection of controls and sample size. We need a multivariate analysis in view of multiple variables.

Our study discovered that recent invasive surgical procedures has an independent association with ESBL/AmpC producing infections. This is in accordance with the studies conducted by Pena $\mathrm{C}$ et al, ${ }^{1}$ Demirdag $\mathrm{K}$ et $\mathrm{al}^{14}$ and Ozgunes $\mathrm{I}$ et $\mathrm{al}^{13}$ who reported surgical intervention as a risk factor. The importance of this factor relates to different epidemiological aspects, such as the effectiveness of infection control measures at the hospital.

The placement of central venous catheter, intravenous catheter and urinary catheter has also been reported as a risk factor in previous studies. ${ }^{1,13}$ Catheter usage may be considered the most common practice at hospitals; therefore the quality of catheter use should be reviewed and improved. The unnecessary use of urinary catheters could be prevented by surveillance and educational interventions. ${ }^{14}$ In the present study, the use of central venous catheters, intravenous catheters and urinary catheters was associated with 3 to 7 times more risk of developing ESBL/AmpC producing infections.

In the 2005 study of Pena $\mathrm{C}$ et al, ${ }^{1}$ female gender and nasogastric tube were identified as independent risk factors. However, our study did not confirm to these findings. Our study also found ICU stay to be an important significant risk factor in acquisition of ESBL/AmpC producing infections.

Revealing risk factors is an important issue for management and control of hospital-acquired infections. ${ }^{14}$ Previous molecular epidemiological studies of ESBL producing E.coli and K.pneumoniae have clearly indicated the nosocomial spread of these isolates. On the basis of these results and the strong association between infection with ESBL-producing E.coli or K.pneumoniae and antibiotic use, we hypothesize that multiple events may be required for infection with ESBL-producing E.coli or K.pneumoniae to develop. First, the patient must acquire ESBLproducing strain through contact with a colonized health care worker or contaminated fomite. Second, the isolate must emerge as a result of the selective effect of 
antibiotic use. These findings have important implications with regard to possible interventions aimed at curbing such outbreaks. Efforts should emphasize limiting contact transmission of resistant isolates as well as controlling antibiotic use. Most interventions aimed at limiting antibiotic use have focussed on restricting the use of extended-spectrum cephalosporins and have met with only modest success. ${ }^{17-19}$

As demonstrated in our study, infection with ESBL-producing E.coli or K.pneumoniae may be related to the use of several different antibiotics, but it is most closely related with total antibiotic use. Elimination of one class of antibiotics, or the substitution of one class for another, without fully addressing the widespread problem of inappropriate antibiotic use will likely result in continued inadequate control of such outbreaks of infection. Emphasis must be placed on the rational and judicious use of all antimicrobial agents. Furthermore, epidemiological studies are the need of the hour that would assess the mechanisms for spread of community acquired multi drug resistant Gram negative bacterial infections, since these mechanisms are currently not understood.

In conclusion, the appropriate antimicrobial use and early removal of unnecessary interventional apparatus is of importance for the control and to decrease the prevalence of ESBL/AmpC -producing organisms. Early identification and avoidance of risk factors is crucial to providing optimal empiric antibiotic therapy to individual patients before the microbiology results are available.

\section{References}

1. Pena C, Gudiol C, Tubau F, Saballs M, Pujol M et al. Risk-factors for acquisition of extended-spectrum $\beta$ lactamase producing Escherichia coli among hospitalised patients. Clin Microbiol Infect 2006;12:279-84.

2. Rupp ME, Fey PD. Extended spectrum beta lactamase producing Enterobacteriaceae. Drugs 2003;63(4):353-65.

3. Baral P, Neupane S, Shrestha B, Ghimire KR, Marasini BP, Lekhak B. Clinical and microbiological observational study on AmpC $\beta$-lactamse-producing Enterobacteriaceae in a hospital of Nepal. Brazilian J Infect Dis 2013;17(2):256-9.

4. Paterson DL, Bonomo RA. Extended spectrum betalactamase: a clinical update. Clin. Microbiol.rev.2005,18(4):657-86.

5. Shanthi M, Sekar U. Extended spectrum beta lactamase producing Escherichia coli and Klebsiella pneumonia: Risk factors for infection and impact of resistance on outcomes. J assoc physicians India 2010;58:41-4.

6. Lee SY, Kotapati S, Kuti JL, Nightingale CH, Nicolau DP. Impact of Extended-Spectrum $\beta$ Lactamase_producing Escherichia coli and Klebsiella species on Clinical Outcomes and Hospital Costs: A Matched Cohort Study. Infect Control Hosp Epidemiol 2006;27:1226-32.

7. Schwaber MJ, Venezia SN, Kaye KS, Ami RB, Schwartz D, Carmeli Y. Clinical and Economic Impact of Bacteremia with Extended-Spectrum $\beta$-LactamaseProducing Enterobacteriaceae. Antimicrobial Agents and Chemotherapy 2006 Apr;50(4):1257-62.
8. Lautenbach E, Patel JB, Bilker WB, Edelstein PH, Fishman NO. Extended-Spectrum $\beta$-LactamaseProducing Escherichia coli and Klebsiella pneumonia: Risk Factors for Infection and Impact of Resistance on Outcomes. Clinical Infectious Diseases 2001 April;32:1162-71.

9. Tumbarello M, Spanu T, Sanguinetti M, Citton R, Montuori E et al. Bloodstream Infections Caused by Extended-Spectrum- $\beta$-Lactamase-Producing Klebsiella pneumonia: Risk Factors, Molecular Epidemiology and Clinical Outcome. Antimicrobial Agents and Chemotherapy 2206 Feb;50(2):498-504.

10. Jacoby GA. AmpC $\beta$-Lactamases. Clinical Microbiology Reviews, 2009 Jan;22(1):161-82.

11. Clinical and Laboratory Standards Institute. Performance standards for antimicrobial susceptibility testing. $21 \mathrm{st}$ Informational Supplement. CLSI document M100-S21 Wayne, PA;2012.

12. Singhal S, Mathur T, Khan S, Upadhyay DJ, Chugh S, Gainnd R, Rattan A. Evaluation of Methods for Amp-C beta lactamases in gram negative clinical isolates from tertiary care hospitals. Indian J Med Microbiol 2005;23:120-4.

13. Ozgunes II, Erben NN, Kiremitci AA, Elif D ED Kartal, Durmaz GG, Colak HH et al. The prevalence of extended spectrum beta lactamase producing Escherichia coli and Klebsiella pneumoniae in clinical isolates and risk factors. Saudi Med J 2006; 27(5):608-12.

14. Demirdag K, Hosoglu S. Epidemiology and risk factors for ESBL-producing Klebsiella pneumonia: a case control study. J Infect Dev Citries 2010;4(11):717-22.

15. Agostini MO, Simor A, Mulvey M, McGeer A, Hirji Z et al. Risk factors for and outcomes associated with clinical isolates of Escherichia coli and Klebsiella species resistant to extended-spectrum cephalosporins among patients admitted to Canadian hospitals. Can J Infect Dis Med Microbiol 2009 Autumn;20(3):e43-8.

16. Du B, Long Y, Liu H, Chen D, Liu D, Xu Y, Xie X. Extended-spectrum beta-lactamase-producing Escherichia coli and Klebsiella pneumoniae bloodstream infection: risk factors and clinical outcome. Intensive Care Med (2002); 28:1718-23.

17. Meyer KS, Urban C, Eagen JA, Berger BJ, Rahal JJ. Nosocomial outbreak of Klebsiella infection resistant to late generation cephalosporins. Ann Intern Med 1993;119:353-8.

18. Rice LB, Willey SH, Papanicolaou GA et al. outbreak of ceftazidime resistance caused bu extended spectrum beta lactamases at a Massachusetts chronic-care facility. Antimicrob Agents Chemother 1990;34:2200-9.

19. Rice LB, Eckstein EC, DeVente J, Shales DM. Ceftaidime resistant Klebsiella pneumoniae isolates recovered at the Cleveland Department of Veterans affairs Medical Center. Clin Infect Dis 1996:23:118-24.

How to cite this article: G. P. Aaftab, B.P. Asha, Medegar S. Multivariate analysis of risk factors for ESBL and AmpC producing Escherichia coli and Klebsiella pneumoniae at a Tertiary Care Hospital in Karnataka: A case control study. Indian J Micribiol Res 2018;5(1):1-6 